\title{
Displaced by deceivers - Prevention of biosensor cross-talk is pivotal for successful biosensor-based high-throughput screening campaigns
}

Lion Konstantin Flachbart ${ }^{1}$, Sascha Sokolowsky ${ }^{1}$ and Jan Marienhagen ${ }^{1,2, *}$

${ }^{1}$ Institute of Bio- and Geosciences, IBG-1: Biotechnology, Forschungszentrum Jülich, D-52425 Jülich, Germany

${ }^{2}$ Institute of Biotechnology, RWTH Aachen University, Worringer Weg 3, D-52074 Aachen, Germany

* To whom correspondence should be addressed.

Tel: +49 246161 2843; Fax: +49 246161 2710; Email: j.marienhagen@fz-juelich.de 
Supplementary Table S1. Strains and plasmids used in this study.

\begin{tabular}{|c|c|c|}
\hline Strain or plasmid & Relevant characteristics & $\begin{array}{l}\text { Reference or } \\
\text { source }\end{array}$ \\
\hline E. coli DH5a & 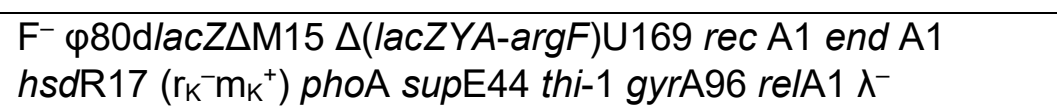 & Invitrogen \\
\hline E. coli TOP10 & $\begin{array}{l}\mathrm{F}^{-} \text {mcrA } \Delta(m r r-h s d \mathrm{RMS}-m c r \mathrm{BC}) \text { } \varphi 80 / a c Z \Delta \mathrm{M} 15 \Delta / a c X 74 \\
\text { recA1 araD139 } \Delta\left(\text { ara-leu) } 7697 \text { galU galK rpsL }\left(\mathrm{Str}^{\mathrm{R}}\right) \text { endA1 }\right. \\
\text { nupG } \lambda^{-}\end{array}$ & Invitrogen \\
\hline E. coli DH10B & $\begin{array}{l}\mathrm{F}^{-} m c r A \Delta(m r r-h s d R M S-m c r B C) \varphi 80 / a c Z \Delta M 15 \Delta / a c X 74 \\
\text { recA1 endA1 araD139 } \Delta(\text { ara, leu }) 7697 \text { gall gal/K } \lambda^{-} r p s L \\
\text { nupG }\end{array}$ & Invitrogen \\
\hline E. coli BL21(DE3) & $\mathrm{F}^{-}$omp $\mathrm{T}$ hsdSB (rB-, mB-) gal dcm rne131 (DE3) & Invitrogen \\
\hline $\begin{array}{l}\text { E. coli DH10B } \\
\Delta h c a R E F C B D\end{array}$ & $\begin{array}{l}\mathrm{F}^{-} \text {mcrA } \Delta(m r r-h s d \mathrm{RMS}-m c r \mathrm{BC}) \varphi 80 / a c Z \Delta \mathrm{M} 15 \Delta / a c X 74 \\
\text { recA1 endA1 araD139 } \Delta\left(\text { ara, leu)7697 galU galK } \lambda^{-} r p s \mathrm{~L}\right. \\
\text { nupG } \triangle \text { hcaREFCBD }\end{array}$ & this work \\
\hline pSIJ8 & $\begin{array}{l}\text { Plasmid enabling L-arabinose inducible expression of } \lambda \text { red } \\
\text { recombineering genes bet, gam and exo. L-rhamnose } \\
\text { inducible expression of flp recombinase gene. }\end{array}$ & (1) \\
\hline pBBR1N-reporter-EYFP & $\begin{array}{l}\text { Biosensor chassis plasmid with the eyfp reporter gene, } \\
\operatorname{Kan}^{R}\end{array}$ & $\begin{array}{l}\text { Markus Schallmey, } \\
\text { unpublished }\end{array}$ \\
\hline pSenCA & $\begin{array}{l}\text { Transcriptional biosensor construct inducing eyfp } \\
\text { expression in response to the presence of trans-cinnamic } \\
\text { acid or phenylpropionic acid, } \text { Kan }^{R}\end{array}$ & This work \\
\hline pBAD/myc-His A & $\begin{array}{l}\text { Cloning vector for the synthesis of } \text { His }_{6} \text {-tagged (c-terminal) } \\
\text { fusion protein, } \mathrm{Amp}^{\mathrm{R}}\end{array}$ & Invitrogen \\
\hline pCBJ296 & pCDFDuet-1 MCS2::xal $T_{T c}, \mathrm{Sp}^{\mathrm{R}}$ & (2) \\
\hline $\mathrm{pBAD}-x a l_{T_{c}}$ & Cloning vector for $\mathrm{Xal}_{\mathrm{Tc}}$ protein synthesis, $\mathrm{Amp}^{\mathrm{R}}$ & This work \\
\hline pBAD-N6XHIS & $\begin{array}{l}\text { Cloning vector for the synthesis of His6-tagged (n-terminal) } \\
\text { protein, Amp }\end{array}$ & This work \\
\hline pBAD-N6XHIS-xal $T_{c}$ & $\begin{array}{l}\text { Cloning vector for the synthesis of His6-tagged (n-terminal) } \\
\mathrm{Xal}_{\mathrm{Tc}} \text { fusion protein, } A \mathrm{mp}^{R}\end{array}$ & This work \\
\hline
\end{tabular}


Supplementary Table S2. Oligonucleotides used in this study. Relevant restriction sites are underlined.

\begin{tabular}{|c|c|c|}
\hline Primer name & Sequence & Resulting plasmid \\
\hline fw_amp_hcaRE' & $\begin{array}{l}\text { AATAATGAGCTCTTATGCCGTTA } \\
\text { CGCTTGCCA }\end{array}$ & pSenCA \\
\hline rv_amp_hcaRE' & $\begin{array}{l}\text { GAGTGAAAGCTTGGCATGCGGT } \\
\text { GTCAATCAGTTGGTAAATGTTC }\end{array}$ & \\
\hline fw_amp_xalTc & $\begin{array}{l}\text { TTCATCGAAACCAATGTTGCAAA } \\
\text { ACC }\end{array}$ & pBAD-xal $T_{T d}$ \\
\hline rv_amp_xalTc & $\begin{array}{l}\text { TTAAAACATTTTACCAACTGCAC } \\
\text { CCATC }\end{array}$ & $\begin{array}{l}\text { PBAD-xal } \\
\text { epPCR library }\end{array}$ \\
\hline fw_amp_pBAD & $\begin{array}{l}\text { CAGTTGGTAAAATGTTTTAAAGA } \\
\text { AGATTTTCAGCCTGATACAGATT } \\
\text { AAATCAG }\end{array}$ & \\
\hline rv_amp_pBAD & $\begin{array}{l}\text { GCAACATTGGTTTCGATGAACAT } \\
\text { GGTTAATTCCTCCTGTTAGCC }\end{array}$ & \\
\hline fw_amp_pBAD_N-6 $\times$ HIS & $\begin{array}{l}\text { CGCAGTCCATGGCGCAGTCTCG } \\
\text { AGCTTGGCTGTTTTGGCGGATG }\end{array}$ & pBAD-N6XHIS \\
\hline rv_amp_pBAD_N-6×HIS & $\begin{array}{l}\text { ACTGCGCCATGGAGTGATGATG } \\
\text { ATGATGATGGCTGCCGCTCATG } \\
\text { GTTAATTCCTCCTGTTAG }\end{array}$ & \\
\hline fw_amp_6xH_xalTc & $\begin{array}{l}\text { ATCATCATCATCATCACTCCATG } \\
\text { TTCATCGAAACCAATGTTGC }\end{array}$ & pBAD-N6XHIS--xal ${ }_{T c}$ \\
\hline rv_amp_6xH_xalTc & $\begin{array}{l}\text { ATCCGCCAAAACAGCCAAGCTT } \\
\text { AAAACATTTTACCAACTGCACCC }\end{array}$ & \\
\hline
\end{tabular}




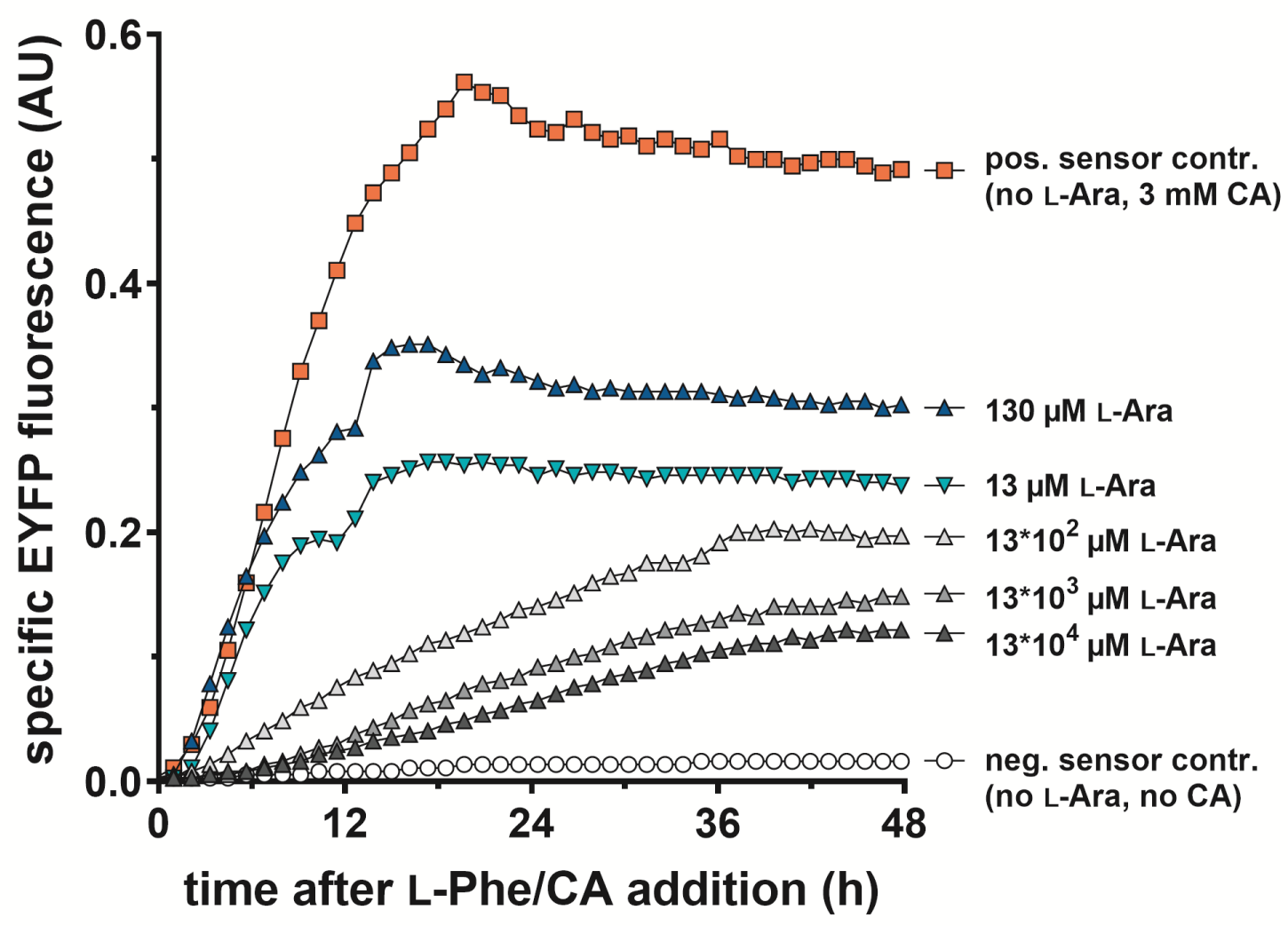

Supplementary Figure S1. Performance of the E. coli pSenCA pBAD-xal Tc $_{c}$ system. After cultivation for $3 \mathrm{~h}$ in presence of the L-arabinose concentrations depicted in the legend, either $3 \mathrm{mM} \mathrm{L-}$ phenylalanine (circles and triangles) or $3 \mathrm{mM} \mathrm{CA}$ (squares) were added. Specific fluorescence response is shown (EYFP fluorescencexbiomass formation ${ }^{-1}$, arbitrary units). Abbreviations: CA, trans-cinnamic acid; L-Ara, L-arabinose; L-Phe, L-phenylalanine. 

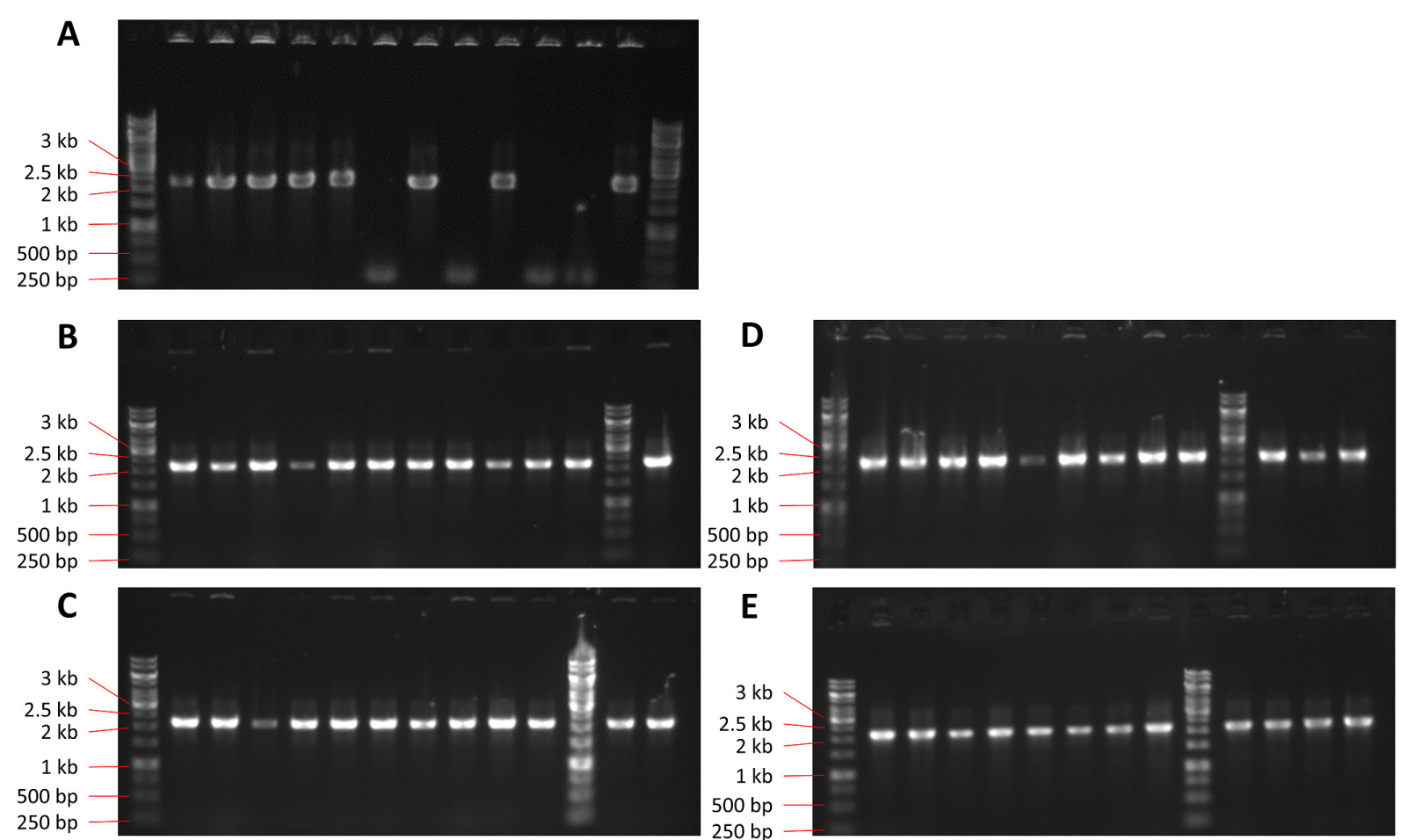

Supplementary Figure S2. Influence of the inoculum size $\left(\mathrm{iOD}_{600}\right)$ on producer isolation efficiency. Overall aim was the identification of suitable assay conditions for the exclusive isolation of E. coli pSenCA pBAD-xal $\left.\right|_{T c}\left(\mathrm{CA}^{+}\right.$-cells) from mixtures with $E$. coli pSenCA pBAD (CA--cells). All cultivations were performed in the in presence of $13 \mu \mathrm{M} \mathrm{L}$-arabinose for induction of heterologous $x a l_{T c}$ expression and $3 \mathrm{mM}$ L-Phe as XAL-substrate was always added three hours after starting each cultivation. Fluorescence-activated cell sorting of the upper $5 \%$ of fluorescent cells was performed five hours after substrate addition. DNA fragments amplified via PCR from isolated cells indicate pBAD (295 $\mathrm{bp})$ or pBADxal $T_{c c}(2,203 \mathrm{bp})$ presence, respectively. (A) Preliminary experiment with an $\mathrm{iOD}_{600}$ of 0.5 and $50 \% / 50 \%$ ratio of $\mathrm{CA}^{+} / \mathrm{CA}^{-}$-cells; (B) iOD 600 of 0.02 and $50 \% / 50 \%$ ratio of $\mathrm{CA}^{+} / \mathrm{CA}^{-}$-cells; (C) $\mathrm{iOD}_{600}$ of 0.004 and $50 \% / 50 \%$ ratio of $\mathrm{CA}^{+} / \mathrm{CA}^{-}-$cells; (D) $\mathrm{iOD}_{600}$ of 0.02 and $20 \% / 80 \%$ ratio of $\mathrm{CA}^{+} / \mathrm{CA}^{-}$-cells; (E) iOD $_{600}$ of 0.004 and $20 \% / 80 \%$ ratio of $\mathrm{CA}^{+} / \mathrm{CA}^{-}$-cells. 
Supplementary Table S3. Overview of all $x a /_{T c}$-variants isolated in the FACS campaign, which were subsequently characterized in detail. Identified mutations are in the open reading frame of $x a l_{T c}$, the $x a T_{T c}$-promoter, $\mathrm{P}_{a}$ aBAD and $\mathrm{P}_{\mathrm{araC}}$, the promoter of the araC gene (encoding the AraC regulator, controlling expression from the $P_{\text {araBAD }}$ promoter), respectively. The araC gene did not carry mutations in any isolated plasmid. Mutations leading to AAS are highlighted in bold. Additionally, the relative CA accumulation of the mutants after isolation and after retransformation into $E$. coli DH10B $\triangle h c a R E F C B D$ is given. Relative CA production after FACS isolation of the respective variants is given as mean of three cultivations and relative CA production after retransformation is given as mean of three biological replicates, the error is depicted as standard deviation. Abbreviations: AAS, Amino acid substitution(s); CA, trans-cinnamic acid.

\begin{tabular}{|c|c|c|c|c|}
\hline mutations in $x a l_{T c}$ & AAS in $\mathrm{Xal}_{\mathrm{Tc}}$ & $\begin{array}{l}\text { Mutations } \\
\text { in } \mathrm{P}_{a r a C} \& \\
\mathrm{P}_{a r a B A D}\end{array}$ & $\begin{array}{l}\text { Relative CA } \\
\text { production } \\
\text { after isolation } \\
\left(\% \mathrm{Xal}_{\mathrm{Tc}}\right)\end{array}$ & $\begin{array}{l}\text { Relative CA } \\
\text { production after } \\
\text { retransformation } \\
\left(\% \text { Xal }_{\mathrm{Tc}}\right)\end{array}$ \\
\hline t363c, t821c & V274A & & $122 \pm 0.8$ & $122 \pm 6$ \\
\hline t570c, a1804g & K602E & & $123 \pm 0.9$ & $115 \pm 5$ \\
\hline $\begin{array}{l}\text { t20c, t1161c, a1391g, } \\
\text { t1812c }\end{array}$ & V7A, N464S & g95a & $123 \pm 0.1$ & $110 \pm 2$ \\
\hline c594t, t821c, a1783t & V274A, N595S & & $123 \pm 0.7$ & $120 \pm 8$ \\
\hline a1725g, a1756g, a1821g & M586V & & $132 \pm 0.5$ & $128 \pm 8$ \\
\hline t821c & V274A & & $134 \pm 1.1$ & $122 \pm 5$ \\
\hline g1731a, a1778t, t1839a & D593V & & $135 \pm 1.3$ & $125 \pm 4$ \\
\hline T499c, t567a, a1806t & F167L, K602N & & $136 \pm 0.8$ & $139 \pm 6$ \\
\hline a250g, t821c, t918c & K84E, V274A & & $137 \pm 1.8$ & $137 \pm 2$ \\
\hline $\begin{array}{l}\text { a45g, a324g, g1365a, } \\
\text { a1391a t1568a t1839a }\end{array}$ & N464S, V523E & & $138 \pm 1.1$ & $136 \pm 5$ \\
\hline & K575R, M586V & & $138 \pm 1.1$ & $123 \pm 6$ \\
\hline g125t, a1185g, t1839c & S42I & $\mathrm{t} 175 \mathrm{a}$ & $139 \pm 0.6$ & $136 \pm 1$ \\
\hline g125t, a1185g, t1839c & S42I & $\mathrm{t} 175 \mathrm{a}$ & $141 \pm 1.6$ & $137 \pm 2$ \\
\hline t987c, t1568a, a1805c & V523E, K602T & & $141 \pm 1.7$ & $132 \pm 4$ \\
\hline t1655c, a1806c & I552T, K602N & & $141 \pm 1.6$ & $131 \pm 5$ \\
\hline t821c, t1836c & V274A & & $143 \pm 0.9$ & $137 \pm 3$ \\
\hline a250g, t821c, t918c & K84E, V274A & & $143 \pm 0.9$ & $129 \pm 4$ \\
\hline $\begin{array}{l}\text { t306c, a1724g, a1756g, } \\
\text { t1839c, t1875c }\end{array}$ & K575R, M586V & & $144 \pm 0.3$ & $113 \pm 4$ \\
\hline t1655a & I552N & a34g & $146 \pm<0.1$ & $159 \pm 2$ \\
\hline $\begin{array}{l}\mathrm{t} 499 \mathrm{c}, \mathrm{t} 1299 \mathrm{c}, \mathrm{a} 1762 \mathrm{c} \\
\mathrm{t} 1839 \mathrm{a}\end{array}$ & F167L, N588H & & $148 \pm 0.7$ & $144 \pm 7$ \\
\hline
\end{tabular}




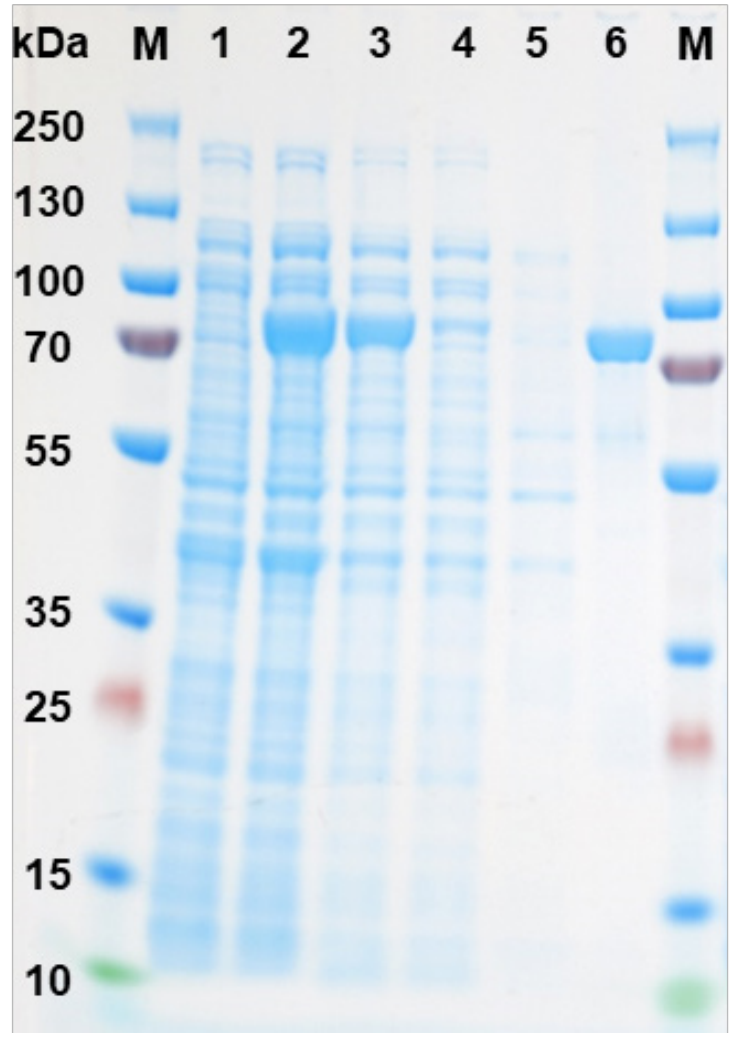

Supplementary Figure S3. SDS-PAGE analysis of a typical Xal $\mathrm{T}_{\mathrm{c}}$ purification conducted for all variants, which were characterized in more detail. Molecular weight of N-terminally His-tagged Xal $\mathrm{Tc}_{\mathrm{T}}$ is $74.8 \mathrm{kDa}$. Abbreviations: M, PAGE Ruler Plus Prestained protein ladder; 1, Whole cells, uninduced heterologous gene expression; 2 , Whole cells, induced heterologous gene expression; 3, Crude cell extract, induced heterologous gene expression; 4, Flow through affinity chromatography; 5, Wash fraction; 6, Elution fraction.

\section{References:}

1. Jensen,S.I., Lennen,R.M., Herrgård,M.J. and Nielsen,A.T. (2015) Seven gene deletions in seven days: Fast generation of Escherichia coli strains tolerant to acetate and osmotic stress. Sci. Rep., 5, 17874.

2. Jendresen,C.B., Stahlhut,S.G., Li,M., Gaspar,P., Siedler,S., Förster,J., Maury,J., Borodina,I. and Nielsen,A.T. (2015) Novel highly active and specific tyrosine ammonialyases from diverse origins enable enhanced production of aromatic compounds in bacteria and yeast. Appl. Environ. Microbiol., 10.1128/AEM.00405-15. 